\title{
Questes
}

Revue pluridisciplinaire d'études médiévales

42 | 2021

Fama : réputation et renommée

\section{La réputation du marchand au Moyen Âge. Un mécanisme informel au service de la sécurité des échanges}

Célia Magras

\section{OpenEdition}

Journals

Édition électronique

URL : https://journals.openedition.org/questes/5741

DOI : $10.4000 /$ questes. 5741

ISSN : 2109-9472

Éditeur

Les Amis de Questes

Édition imprimée

Date de publication : 28 janvier 2021

Pagination : 109-122

ISSN : 2102-7188

Référence électronique

Célia Magras, «La réputation du marchand au Moyen Âge. Un mécanisme informel au service de la sécurité des échanges », Questes [En ligne], 42 | 2021, mis en ligne le 28 février 2021, consulté le 05 février 2022. URL : http://journals.openedition.org/questes/5741 ; DOI : https://doi.org/10.4000/ questes. 5741 


\section{La réputation du marchand au Moyen Âge. Un mécanisme informel au service de la sécurité des échanges.}

\section{Célia MAgras}

Université de Bordeaux

«Si tu pratiques le commerce ou tiens une boutique, garde tes yeux grands ouverts, nuit et jour [...]. Ne te fie pas à n'importe qui ${ }^{1} »$.

Dans le monde des affaires, le regard porté par les autres est fondamental ${ }^{2}$ car il permet de forger la réputation. C'est un " gran tesoro $^{3}$ », parfois plus précieux que l'argent. Qu'importe le lieu, les marchands médiévaux sont toujours « anxieux d'être perçus comme honnêtes, car leur bonne réputation les précède lorsqu'ils désirent échanger avec les autres commerçants ${ }^{4} »$. Le lien d'affaires ne repose pas toujours sur une relation personnelle de proximité. Il doit répondre à des règles spécifiques, afin de suppléer le danger que peut représenter l'absence d'informations directes permettant de forger la confiance.

\footnotetext{
${ }^{1}$ Traduction d'un poème en dialecte génois d'un auteur italien inconnu de la fin du XIII ${ }^{\mathrm{e}}$ siècle. Robert Sabatino Lopez, «Le marchand génois : un profil collectif», Annales. Économies, sociétés, civilisations, $13^{\mathrm{e}}$ année, $\mathrm{n}^{\circ} 3,1958, \mathrm{p} .501-515$.

${ }^{2}$ Paul Milgrom, Douglass North et Barry Weingast, « The Role of Institutions in the Revival of Trade », Economics and Politics, vol. 2, n 1, March 1990, p. 123.

3 « Meglio è a l'uomo avere buona fama in questo mondo che avere un gran Tesoro », Paoloda Certaldo (Un marchand italien du début du XIV ${ }^{\mathrm{e}}$ siècle), Il libro di buoni costumi, F. Le Monnir, 1945, p. 62.

4 Emily Kadens, «The Medieval Law Merchant: The Tyranny of a Construct», December 2018: $\quad$ https://brewminate.com/the-medieval-law-merchant-the-tyranny-of-aconstruct/, consulté le 8 octobre 2019.
} 
Pour saisir la notion de confiance, il faut préciser d'emblée qu'il ne s'agit pas seulement d'une donnée psychologique : elle n'est pas offerte, mais doit être gagnée. Dans le domaine économique, elle est le fruit d'un «calcul rationnel visant à déterminer ce que nous pouvons attendre d'un autre acteur ${ }^{5} »$. Un tel calcul recouvre tant d'éléments différents qu'il paraît difficile à établir, et les notions de crédit et de confiance semblent presque insaisissables parce qu'elles se trouvent au carrefour de toutes les disciplines. Néanmoins, dans le commerce, le prisme de la réputation permet de saisir la confiance en s'échappant du domaine psychologique, car elle lui sert de vecteur.

La réputation se trouve tout entière dans le regard que porte la société sur une personne, déterminant une perception qui peut être favorable ou défavorable à son sujet. Elle se constitue de la totalité des jugements portés par un groupe social dont l'importance varie. Ils façonnent cette "matière psychique collective ${ }^{6}$ » à partir d'un ensemble d'éléments observables qui autorisent l'évaluation du marchand. La matière qui permet d'établir la réputation est difficile à définir avec précision puisqu'elle se constitue de manière indirecte d'après l'intégralité des comportements considérés par l'opinion ${ }^{7}$. Il semble impossible de présenter exhaustivement les données qui contribuent à la genèse d'une bonne réputation. Néanmoins, l'étude restreinte au cadre commercial légitime la focalisation de notre attention sur un groupe déterminé. De plus, les besoins des hommes d'affaires sont spécifiques et la réputation est instrumentalisée pour y répondre efficacement.

Nous allons donc tenter d'en dresser un tableau aussi fidèle que possible, afin de percevoir les actions ainsi que les traits de personnalité qui rendent un marchand digne de confiance et qui varient selon les époques. Le contexte en ce

\footnotetext{
${ }^{5}$ Timothy Guinnane, «Les économistes, le crédit et la confiance », Genèses, n $79,2010 / 2$, p. 625.

${ }^{6}$ Lucien Karpik, « Réalité marchande et réputation », Communication, n ${ }^{93}, 2013$, p. 121129.

${ }^{7}$ Nelson Polsby, Community Power and Political Theory, New Haven, Yale University Press, 1963, p. 53.
} 
domaine est fort important. La réputation, en tant que mécanisme informel, doit répondre aux nécessités pratiques du commerce et évoluer en même temps que lui. Si l'obligation de disposer d'une bonne réputation demeure inchangée, les critères pris en considération, de même que les modalités de transmission de l'information, sont, quant à eux, dépendants des besoins du moment.

L'intérêt majeur d'une étude de la réputation dans le monde des affaires est qu'elle ne s'embarrasse pas des frontières. Les archives médiévales italiennes, françaises et égyptiennes mettent en lumière l'importance cruciale de ce mécanisme dans la communauté commerçante à travers deux questions fondamentales. Comment la construire ? Et comment la maintenir ${ }^{8}$ ? Qu'importe l'origine du marchand, les informations prises en considération pour estimer la valeur d'un partenaire commercial sont les mêmes.

Le comportement professionnel de l'individu est aussi important que son comportement personnel. Contrairement à ce qu'affirme l'allocution homo mercator vix aut numquam potest Deo placere ${ }^{9}$ datée des années $1140-1150^{10}$, le cadre moral imposé entre commerçants est très empreint de théologie. La réputation est le reflet de sa capacité à se conformer à ces préceptes, elle conditionne le lien entre les marchands à tous les niveaux. Refuser de s'y conformer, c'est risquer l'ostracisation, et donc la paralysie professionnelle.

\section{La réputation comme imputation : un mécanisme d'évaluation}

\footnotetext{
${ }^{8}$ Jessica Golberg, Trade and Institutions in the Medieval Mediterranean: The Geniza Merchants, Cambridge, Cambridge University Press, 2012, p. 146.

${ }^{9}$ «Le commerçant peut agir sans pécher, mais il ne peut pas être agréable à Dieu ».

${ }^{10}$ Voir à ce sujet André Vauchez, "Homo mercator vix aut numquam potest Deo placere": quelques réflexions sur l'attitude des milieux ecclésiastiques face aux nouvelles formes de l'activité économique au XII ${ }^{\mathrm{e}}$ siècle et au début du XIII ${ }^{\mathrm{e}}$ siècle », dans Le marchand au Moyen Âge, Actes du XIX ${ }^{e}$ congrès de la Société des historiens médiévistes de l'enseignement supérieur public, Paris, SHMES, 1992, p. 211-217.
} 
Les transactions commerciales se réalisent sur de très grandes distances et les informations circulent souvent trop lentement ${ }^{11}$ pour le rythme des affaires. L'évaluation efficace de ses partenaires financiers est donc plus nécessaire que jamais ${ }^{12}$. Elle repose en grande partie sur la réputation qui permet de savoir comment appréhender un acteur sans disposer de connaissances précises à son sujet. Il s'agit d'une forme de vitrine pour le commerçant qui l'autorise ou non à être reconnu par ses partenaires commerciaux comme un sujet digne de confiance $^{13}$.

Pour comprendre la consistance de la réputation commerciale, il faut avant tout avoir à l'esprit qu'elle sert des intérêts économiques pragmatiques. Les premiers inventaires de la langue française mettent directement en lumière l'existence d'un lien entre le crédit et la réputation. Bien qu'il définisse le sens du terme réputation en donnant des traductions latines, le Trésor de la langue francoyse tant ancienne que moderne de 1606 opère tout de même ce parallèle : «avoir bon crédit, \& bonne réputation, être bien estimé ${ }^{14} »$. Un rapprochement plus direct a lieu moins d'un siècle plus tard dans la première édition du Dictionnaire de l'Académie française en 1694 à propos du terme crédit: «Réputation où l'on est de bien payer, et qui est cause qu'on trouve aisément à emprunter ${ }^{15} »$.

Sans équivoque, la réputation représente un intérêt financier et permet de faciliter les échanges d'argent. Rien d'étonnant à cela puisque le latin reputatio

\footnotetext{
${ }^{11}$ Bernard Doumerc, «Par Dieu écrivez plus souvent ! La lettre d'affaires à Venise à la fin du Moyen Âge », La Circulation des nouvelles au Moyen Âge, XXIV ${ }^{\mathrm{e}}$ Congrès de la S.H.M.E.S., Rome, École Française de Rome, 1994.

${ }^{12}$ Gunnar Dahl, Trade, Trust, and Networks. Commercial Culture in Late Medieval Italy, Lund, Nordic Academic Press, 1998, p. 298.

13 Giacomo Todeschini, "Theological Roots of the Medieval/Modern Merchants' SelfRepresentation », dans The Self-Perception of Early Modern "Capitalists", dir. Margaret C. Jacob et Catherine Secretan, New York, Palgrave MacMillan, 2008, p. 10.

${ }^{14}$ Jean Nicot, Trésor de la langue francoyse tant ancienne que moderne, Paris, D. Douver, 1606, p. 165.

${ }_{15}$ Dictionnaire de l'academie francoise dédié au roi, Paris, Veuve de Jean Baptiste Coignard, 1694, t. I, p. 289.
} 
renvoie à l'idée d'évaluation. Or, évaluer son partenaire commercial avant de s'engager est un impératif pour protéger la sécurité des échanges ${ }^{16}$. La confiance représente de l'argent puisqu'elle sert à mesurer sans cesse les risques et les avantages. Il s'agit d'une garantie à laquelle peut recourir le créancier pour apprécier les risques que représente celui envers lequel il s'engage dans une relation financière. En témoigne le double sens du terme «crédit », qui est à la fois une notion objective (l'actif dont on dispose) et une notion subjective (la confiance que l'on accorde) $)^{17}$.

Elle ne permet pas seulement de jauger la personne, mais surtout d'estimer les risques financiers qu'elle pourrait représenter sans la connaître personnellement. Finalement, la réputation est une imputation. De très nombreuses informations sont donc prises en considération. Pour avoir une bonne réputation, le marchand doit disposer d'un « état de dignité intact approuvé par les mœurs et les lois, et qui n'est amoindri en aucun point ${ }^{18} »$. Aussi l'ensemble de son comportement personnel ou professionnel est-il constamment évalué. L'éthique marchande a pour source la morale générale avec laquelle elle fusionne, puisque le commerçant est en partie jugé sur les mêmes données que les autres hommes.

$\mathrm{Du}$ fait de son importance cardinale dans les affaires, le thème est omniprésent dans les écrits de commerçants, dans leurs correspondances, leurs contrats ou dans les manuels de commerce qui fleurissent au $\mathrm{XV}^{\mathrm{e}}$ siècle $^{19}$. Les incursions dans les archives de cette communauté mettent en lumière les éléments considérés comme utiles pour décider si un partenaire commercial potentiel est

\footnotetext{
${ }^{16}$ Cécile Kharoubi et Philippe Thomas, Analyse du risque de crédit, Banque \& marchés, Paris, RB éditions, 2016.

${ }^{17}$ Voir à ce sujet Émile Benveniste, Le vocabulaire des institutions indo-européennes, Paris, Les Éditions de Minuit, 1969, t. 1, p. 172.

${ }^{18}$ Anne Grondeux, «Le vocabulaire de la renommée au Moyen Âge », Médiévales, $\mathrm{n}^{\circ} 24$, 1993, p. 17.

${ }^{19}$ Robert Sabatino Lopez, «Un texte inédit: le plus ancien manuel italien de technique commerciale », Revue historique, Paris, 1970, p. 67.
} 
digne de confiance ${ }^{20}$. Dans ce cadre, un très grand nombre d'éléments sont pris en considération car ils représentent autant d'indices qui permettent de s'assurer que la confiance offerte aux partenaires commerciaux est méritée.

Le contrat d'engagement conclu entre les frères Salimbeni de Sienne en $1280^{21}$ met en exergue la manière dont la réputation encadre le comportement d'un nouveau partenaire commercial. La nouvelle recrue s'engage, en jurant sur le livre des Évangiles, à éviter toute forme de tromperie économique qui pourrait nuire directement à la confiance accordée à la société, mais aussi à se comporter chaque jour en bon chrétien. Cela suppose qu'elle évite tous les actes contraires aux bonnes mœurs, comme le jeu d'argent ou l'adultère. On trouve des éléments similaires dans les archives de la Genizah du Caire, et les correspondances entre commerçants définissent clairement le comportement attendu d'un partenaire commercial : «agir pour le bien commun, éviter la tromperie, la négligence et le laxisme et agir comme une personne pieuse et un gentleman ${ }^{22} »$, mais aussi ne «pas mettre son propre intérêt avant celui de ses associés ${ }^{23}$ ».

Le comportement personnel et professionnel du commerçant doit être irréprochable ${ }^{24}$. Francesco di Balduccio Pegolotti, un homme d'affaires florentin $\mathrm{du} \mathrm{XIV}^{\mathrm{e}}$ siècle, résume les critères pris en considération dans son manuel de commerce $^{25}$. Il reprend les vers de Dino Compagni, un historien chroniqueur et auteur italien du Trecento, d'après lesquels le marchand doit agir selon l'équité, être d'aspect affable et fréquenter l’Église ${ }^{26}$. Pour avoir bonne réputation aux yeux

20 Sheilagh Ogilvie, Institutions and European Trade: Merchant Guilds, 1000-1800, Cambridge, Cambridge University Press, p. 318.

${ }^{21}$ Armando Sapori, Studi di storia economica, Firenze, Sansoni, 1955, p. 762-763.

${ }^{22}$ TS 8 J 11, f, 14, ligne 12 à 13, traduction de l'anglais « to exert himselffor the common good, to shun cheating, negligence and easy going, to act as a pious person and a gentleman », Shelemo Dov Goiten, "Commercial and Family Partnerships in the Countries of Medieval Islam », Islamic Studies, vol. 3, n 3, 1964, p. 315-337.

23 Ibid.

${ }^{24}$ Jacques Le Goff, Marchands et banquiers du Moyen Âge, Paris, PUF, 2001, p. 84.

${ }^{25}$ Franceso Balducci Pegolotti, La Pratica della mercatura [1335-1343], trad. Allan Evans, Cambridge, The Mediaeval Academy of America, 1936.

${ }^{26}$ Traduction citée par $i d$. 
de la société, mais aussi de ses pairs, le commerçant ne peut se contenter de respecter ses engagements, il doit traduire « dans ses conduites l'intériorisation de valeurs morales ${ }^{27} \gg$.

Au Moyen Âge, savoir à qui faire confiance repose tout autant sur la vie privée que sur le comportement commercial de l'agent. L'étude menée par Daniel Lord Smail sur les archives des procès pour dettes marseillais entre 1264 et 1423 permet de saisir avec encore plus de précision la consistance de la notion de réputation dans le cadre des échanges ${ }^{28}$. Lorsqu'aucune donnée écrite ne justifie l'existence d'une dette, un vendeur peut toujours invoquer le fait d'être honnête et mener une vie sans tache, à condition de trouver des témoins pour en attester $^{29}$. Le commerçant sera jugé sur un ensemble de données loin de se cantonner au seul domaine économique : sa conduite privée permet d'obtenir des informations sur sa moralité. S'il ne joue pas, agit en bon chrétien ou, mieux encore, s'il se montre généreux envers les plus démunis, il laissera présager un bon comportement économique ${ }^{30}$. D'ailleurs, lors de la signature des contrats, il est fréquent de prendre Dieu pour témoin et de lui verser, en contrepartie, une somme d'argent ${ }^{31}$.

Un dernier élément est pris en considération afin d'estimer, grâce au comportement passé de l'agent ${ }^{32}$, les risques qu'il pourrait représenter dans le futur : sa capacité à honorer ses engagements. Cet élément revêt logiquement une importance considérable dans l'optique d'une évaluation du risque que matérialise un potentiel partenaire commercial. Lorsqu'un commerçant ne paie pas ses dettes, il brise le lien de réciprocité et de confiance qui l'unit à ses pairs.

27 Laurence Fontaine, L'économie morale, Mayenne, Gallimard, 2008, p. 277-307, Chapitre X : «Construire la confiance », cit. p. 284.

${ }^{28}$ Ibid., p. 133 et suivantes.

${ }^{29}$ Voir par exemple le cas de Jacma Thomasia, une vendeuse de vin ou une tavernière dans le cadre d'un procès pour dette initié en 1339, ADBR 3B 37, fols. 264r-269r, cité par Daniel Lord Smail, The Consumption of Justice, London, Cornell University Press, 2003, p. 151.

${ }^{30}$ Laurence Fontaine, L'économie morale, op. cit.

${ }^{31}$ Jacques Le Goff, Marchands et banquiers du Moyen Âge, op. cit., p. 85.

${ }^{32}$ Jessica Golberg, Trade and Institutions in the Medieval Mediterranean..., op. cit., p. 169. 
Du statut d'individu honorable à celui d'éternel suspect, le chemin est court, puisqu'il suffit de ne pas rembourser un créancier.

Cette importance de l'exactitude des paiements se retrouve dans de très nombreux écrits de marchands médiévaux. Le premier manuel consacré à la pratique commerciale, Della mercatura e del mercante perfetto, rédigé par le marchand italien Bennedetto Cotrugli aux alentours de 1458, est traduit en français dès $1582^{33}$. Tout en déconseillant la vente à terme, il présente néanmoins certains critères à prendre en considération pour limiter les risques. Il requiert de privilégier un partenaire commercial « de bonne renommée, qui ait bon crédit, qu'il soit de bon entendement, et bon payeur ${ }^{34} »$.

On retrouve cette importance de l'exactitude des paiements plus loin dans un passage consacré à « [...] la manière de payer la dette ${ }^{35}$ », où il affirme qu' « en bien payant on acquiert bonne renommée et crédit envers les amis ${ }^{36} »$. Les relations commerciales dépendent de la manière dont le marchand est perçu par ceux qui font fructifier son affaire. La réputation permet d'associer à son nom une représentation communautaire partagée issue de son évaluation sociale ${ }^{37}$, ce qui peut lui servir de garantie lorsqu'il respecte les règles de conduite prescrites.

En revanche, le risque pour celui qui ne se conforme pas à ces préceptes encadrant la vie personnelle et professionnelle se trouve directement dans la remise en cause de la réputation. Il ne s'agit pas seulement d'un instrument d'appréciation, mais aussi d'une sanction économique. La réputation est aussi précieuse qu'elle est fragile. Lorsque les comportements du marchand s'éloignent

\footnotetext{
${ }^{33}$ Jean Boyron, Traicté de la marchandise et du parfait marchand en IV livre, Lyon, 1582. Un ouvrage réédité récemment par Luc Marco et Robert Noumen, Traité de la marchandise et du parfait marchand, Paris, L'Harmattan, 2008.

${ }^{34}$ Ibid., p. 40.

${ }^{35}$ Ibid., p. 60.

${ }^{36}$ Ibid., p. 62.

${ }^{37}$ Pierre-Marie Chauvin, «La sociologie des réputations. Une définition et cinq questions », Communications, vol. 93, $\mathrm{n}^{\circ}$ 2, 2013, p. 131-145.
} 
du cadre moral imposé par ses pairs, il risque l'ostracisation, et donc la paralysie commerciale.

\section{La réputation comme sanction : un mécanisme d'ostracisation}

Tout comme dans la société civile, la réputation conditionne l'appartenance au corps social, mais elle conditionne aussi l'intégration dans la communauté commerçante. C'est sur elle que repose la promotion de comportements honnêtes dans un groupe où la confiance ne repose pas toujours sur une relation de proximité ${ }^{38}$. Qu'importe le commerce, qu'il soit d'argent ou de bien, qu'il soit sédentaire ou itinérant, il suppose toujours que des relations d'échanges s'établissent. Or, une mauvaise réputation paralyse totalement l'activité puisque «les gens ne peuvent fonctionner sans les gens ${ }^{39} »$. La sanction d'un agent dont la réputation est remise en cause, soit que sa moralité soit soumise à caution, soit qu'il se soit mal comporté dans ses affaires, est bien plus rapide que celle mise en place par les tribunaux ${ }^{40}$, puisqu'elle rompt le lien de confiance qui unit les commerçants entre eux.

Un marchand florentin anonyme du XIV siècle, lorsqu'il résume les conditions essentielles pour réussir dans les affaires, prescrit de ne pas se lier à ceux qui ont mauvaise réputation ${ }^{41}$. Cette nécessité pratique se manifeste directement dans les contrats d'alliances entre les commerçants. Le contrat de la famille Salimbeni cité précédemment ${ }^{42}$ en fait la condition sine qua non de

\footnotetext{
${ }^{38}$ Paul Milgrom, Douglass North et Barry Weingast, « The Role of Institutions in the Revival of Trade », art. cit.

${ }^{39}$ Traduction de l'anglais, " people cannot operate without people », Lettre d'un marchand, Genizah du Caire, DK 22, b, 1. 18, Avner Greif, «Reputation and Coalitions in Medieval Trade : Evidence on the Maghribi Traders », The Journal of Economic History, vol. 49, n 4 , p. 864.

${ }^{40}$ Ibid.

${ }^{41}$ «Non si vuole pigliare l'amistà di ognuno e ispeziale di quelli che non sono abienti o che non sono di netta fama ». Corti Gino, «Consigli Sulla Mercatura Di Un Anonimo Trecentista », Archivio Storico Italiano, vol. 110, $\mathrm{n}^{\circ} 1,1952$, p. 114-119.

${ }^{42}$ Ibid.
} 
l'engagement d'un nouveau partenaire. Ce critère décisif de l'alliance économique se retrouve aussi dans les archives de la Genizah du Caire, où un marchand requiert que son partenaire commercial soit « digne de confiance devant une cour de justice ${ }^{43} \gg$. Il s'agit d'un point crucial pour conclure un partenariat ou une commenda. De même, le manuel de Benedetto Cotrugli affirme qu'il faut absolument éviter de faire crédit aux individus qui ont mauvaise réputation, car cela suppose de courir un risque ${ }^{44}$.

Elle sert donc de renfort aux relations entre les marchands afin de limiter les possibilités de trahison ${ }^{45}$. Elle est un outil qui répond à un besoin fondamental pour encadrer le commerce à longue distance ${ }^{46}$. Les conséquences de sa perte sont tellement efficaces qu'elles permettent de compenser la faiblesse des mécanismes institutionnels d'encadrement du crédit ${ }^{47}$. Sans cadre légal sûr ${ }^{48}$, avec des moyens de communication limités et souvent très lents, les marchands ont besoin de trouver des façons de protéger leurs engagements et leurs contrats ${ }^{49}$. L'ostracisation de celui qui s'écarte du droit chemin est rapide et sans appel, et n'a pas besoin d'un relais institutionnel pour sanctionner ceux qui ne respectent pas les règles prescrites.

Paul R. Milgrom, Douglass C. North et Barry R. Weingast s'interrogent même sur l'utilité des gardes de foires, puisque les commerçants sont, dans une

\footnotetext{
${ }^{43}$ Une expression traduite de l'hébreu. Voir Shelemo Dov Goiten, « Commercial and Family Partnerships in the Countries of Medieval Islam », art. cit., p. 320.

44 Jean Boyron, «De la vente qui se fait à terme », p. 40.

45 «Celebrating sociability and circulated information on other members reputation clearly had the function of strengthened mutual relationship in order to make cheating with the network more difficult », dans Christian Ewert et Stephan Selzer, Institution of Hanseatic Trade, Studies on the Political Economy of a Medieval Network Organisation, Peter Lang, 2017, p. 20.

46 Giacomo Todeschini, "Theological Roots of the Medieval/Modern Merchants' SelfRepresentation », art. cit., p. 17-46.

${ }^{47}$ Daniel Lord Smail, The Consumption of Justice, op. cit., p. 149.

${ }^{48}$ Voir Claude Dupouy, Le droit des faillites en France avant le code de commerce, Paris, Librairie générale de droit et de jurisprudence, 1960, et Célia Magras, La constance des stigmates de la faillite : de l'Antiquité à nos jours, Thèse soutenue à l'Université de Bordeaux, LGDJ, à paraître.

${ }^{49}$ Emily Kaden, « The Medieval Law Merchant : The tyranny of a construct », art. cit.
} 
grande mesure, capables de s'autogérer par le biais de mécanismes informels d'exclusion des agents défaillants ${ }^{50}$. L'étude de la réputation dans le monde des affaires au Moyen Âge permet ainsi d'observer la manière dont une communauté s'est organisée pour mettre en place des sanctions plus efficaces que celles instaurées par les pouvoirs publics dans le but de suppléer aux difficultés institutionnelles de l'époque ${ }^{51}$.

En dehors de la simple exclusion a posteriori des individus qui ne respectent pas les règles de comportement prescrites par le groupe, elle offre une garantie a priori de la bonne conduite des échanges. Elle permet de rendre le gain qui résulte d'une tromperie moins intéressant que la perte qu'il risque de produire s'il vient à la connaissance des partenaires commerciaux. Le bénéfice à court terme ne peut en effet pas, ou rarement, être suffisant pour justifier la paralysie professionnelle qu'il génère à long terme ${ }^{52}$. La réputation est donc un mécanisme endogène parfaitement indépendant qui se trouve entre les mains des commerçants. Grâce à elle, ils disposent d'un pouvoir de vie et de mort économique sur leurs semblables.

La moindre rumeur, le plus petit élément qui permettrait de remettre en cause la moralité d'un marchand peut être lourd de conséquences sur sa carrière. On remarque néanmoins de la part de certains commerçants une volonté de s'assurer de la véracité des propos rapportés à propos du mauvais comportement d'un agent économique avant de remettre en cause sa réputation. Le marchand Abraham ben Yiygu requiert par exemple à son correspondant de ne rien dire à personne $e^{53}$ jusqu'à avoir acquis la certitude qu'il avait « dévié du droit chemin ${ }^{54}$ ».

\footnotetext{
${ }^{50}$ Paul Milgrom, Douglass North et Barry Weingast, art. cit., p. 1-23.

${ }^{51}$ Jessica Golberg, Trade and Institutions in the Medieval Mediterranean..., op. cit., p. 170171.

${ }^{52}$ Paul Milgrom, Douglass North et Barry Weingast, art. cit.; Avner Greif, « Reputation and Coalitions in Medieval Trade : Evidence on the Maghribi Traders », art. cit.

${ }^{53}$ Letter from Yosef b. Avraham to Avraham b. Yiyju (1136-1139), «let no one know », TS 10J12.5 + TS NS J181 + TS AS 146.12, trad. Shelemo Dov Goiten, India Traders of the Middle Ages : Documents from the Cairo Geniza, Leyden, Brill, 2008, p. 578.

${ }^{54}$ Ibid.
} 
La réputation en matière commerciale constitue la seule protection humaine contre les risques liés aux relations de crédit. Elle se trouve «au cœur des arrangements mis en place par les marchands pour assurer la bonne foi des échanges ${ }^{55}$ », non pas en raison de considérations morales - ne pas payer est mal mais en raison de considérations pratiques. Toute l'efficacité de la réputation des marchands en tant que garantie économique repose néanmoins sur un seul postulat : celui que les agents considérés du fait de leur mauvaise réputation comme dangereux seront exclus. La confiance n'est pas une donnée stable, elle n'est jamais définitivement acquise et nécessite un entretien constant et appliqué $^{56}$, car elle repose sur la réputation. Elle peut faire et défaire des avenirs professionnels.

Parce que la réputation est immatérielle, qu'elle se nourrit de très nombreux critères, les dégâts que peuvent causer sa remise en cause sont considérables. Il suffit parfois d'une accusation sur l'existence d'un comportement critiquable ou d'un impayé, qu'elle soit ou non fondée, pour fragiliser la fama et déstabiliser une carrière, d'autant plus si elle est débutante. Le risque que représente la rumeur d'une créance non remboursée pour la réputation d'un débiteur apparaît considérable. Une simple insulte peut se transformer et s'étendre jusqu'à pousser les créanciers à douter de la solvabilité d'un débiteur ${ }^{57}$. Les vecteurs de l'information sont multiples. Elle représente «un atout primordial dans les affaires $^{58} »$.

D'ailleurs, les modes judiciaires de recouvrement des créances ne sont utilisés qu'en dernier recours ${ }^{59}$ à cette époque. Le simple fait de réclamer

\footnotetext{
${ }^{55}$ Karim Benyekhlef, Une possible histoire de la norme : les normativités émergentes de la mondialisation, Montréal, Thémis, 2008, p. 474.

${ }^{56}$ Robert Damien, « La confiance et ses crises philosophiques », dans La confiance en question, dir. Romain Laufer et Magali Orillard, Paris, L'Harmattan, 2000, p. 19-36.

${ }^{57}$ Laurence Fontaine, L'économie morale, op. cit., p. 285.

${ }^{58}$ Bernard Doumerc, «Par Dieu écrivez plus souvent! La lettre d'affaires à Venise à la fin du Moyen Âge », art. cit., p. 99-109.

${ }^{59}$ Daniel Lord Smail, The Consumption of Justice, op. cit., p. 148-150.
} 
publiquement le remboursement d'une dette s'interprète à lui seul comme une insulte ou une agression particulièrement humiliante pour le présumé débiteur ${ }^{60}$. Lorsque l'information d'une défaillance se transmet, l'avenir commercial est plus qu'incertain. Aussi la publicité, parce qu'elle est néfaste à la réputation, constituera-t-elle l'une des armes favorites de la gestion coutumière puis légale de l'insolvabilité, puisqu'elle permet d'ostraciser le débiteur inconséquent ${ }^{61}$.

Les correspondances de la Genizah du Caire matérialisent la violence sociale et professionnelle d'un procès pour dette ${ }^{62}:$ «les gens sont devenus anxieux et hostiles avec moi et tous ceux qui me devaient de l'argent conspirent pour ne pas me le rendre ${ }^{63} »$. Les conséquences de cette accusation sont si violentes que celui dont la réputation est remise en cause pense à « quitter ce monde ${ }^{64} \gg$. Dans la société médiévale, le fait pour un créancier de s'en remettre aux tribunaux pour réclamer sa dette est le témoignage direct d'une crise de la confiance. C'est un acte particulièrement grave, car il donne à la défaillance une publicité considérable qui facilite la transmission de l'information et l'ostracisation qui en découle.

La réputation dans les affaires est un bien précieux qu'il faut construire, entretenir et protéger constamment, l'un des seuls sur lesquels l'ensemble des hommes peut se reposer au Moyen Âge. Elle offre une forme de crédit moral parfois bien plus important que la solvabilité réelle pour assurer la pérennité d'une relation commerciale. Sans elle, aucune chance pour le marchand de prospérer

\footnotetext{
${ }^{60}$ Ibid., p. 148.

${ }^{61}$ Célia Magras, «L'instrumentalisation de l'humiliation en droit de la faillite : un mécanisme d'ostracisme commercial », dans L'humiliation. Discours, représentations et pratiques, à paraitre.

62 «I was accused of things which I had no knowledge and which I did not commit», Bodl. Heb. A2/17, Lettre datée de septembre 1040 envoyée par alMahdiyya by Yahya b. Musa alMajjani à Zechariah b. Tammam à propos d'un procès pour dette. Trad. Shelemo Dov Goiten, India Traders of the Middle Ages: Documents from the Cairo Geniza, op. cit., p. 104.

${ }^{63}$ Ibid.

${ }^{64}$ Ibid.
} 
dans les affaires ; ni ses pairs ni ses potentiels clients n'offriront leur confiance de manière durable et il se trouvera exclu du tissu des échanges. Si l'importance des préceptes religieux dans le comportement du commerçant a grandement diminué avec les siècles, celle de la réputation n'a pas changé. Aujourd'hui encore, elle sert à la fois de mécanisme d'évaluation et d'ostracisation des agents économiques. 